\title{
Aspectos biológicos de las rayas Dasyatis guttata y Dasyatis americana (Myliobatiformes: Dasyatidae) capturadas por la pesquería artesanal de la Isla de Margarita, Venezuela
}

\author{
Biological aspects of rays Dasyatis guttata and Dasyatis americana (Myliobatiformes: Dasyatidae) \\ caught by the artisanal fishery in Margarita Island, Venezuela
}

\begin{abstract}
Alejandro Tagliafico ${ }^{1}$, Néstor Rago ${ }^{2}$ y María Salomé Rangel ${ }^{1,3}$
${ }^{1}$ Escuela de Ciencias Aplicadas del Mar, Núcleo de Nueva Esparta, Universidad de Oriente, Boca de Río, 6304, Isla de Margarita, Venezuela. tagliaficoa@gmail.com

${ }^{2}$ Oceanografía y Pesca, Fundación de Ciencias Naturales La Salle, Isla de Margarita, Venezuela. nestorrago@yahoo.es ${ }^{3}$ Área de Biología, Departamento de Ciencias, Núcleo de Nueva Esparta, Universidad de Oriente, Guatamare, 6311, Isla de Margarita, Venezuela. salome453@gmail.com

Abstract.- Dasyatis guttata and D. americana are target species of some artisanal fisheries in Venezuela. Because there are directed fisheries for their catches and there have been few studies on its biology, both species are listed in the IUCN as Data Deficient. Therefore, the objectives of this research were to analyze the catches of the artisanal fishery in Margarita Island in order to provide reproductive data to contribute to the management of both species. For this reason, 3 fishing communities and 2 markets selling fish were sampled once a week, from January 2006 to December 2007. Disc width, sex and maturity were determined for each specimen captured. A total of 355 specimens of $D$. guttata showed that females $(32-173 \mathrm{~cm})$ were larger than males $(28.6-113 \mathrm{~cm}), 35 \%$ of the catches were immature individuals, and although exists differences in the sex ratio there were no difference in the size at maturity $\left(\mathrm{L}_{50}\right.$ to both sexes: $56 \mathrm{~cm}$ ); nonetheless, all individuals were mature above $78 \mathrm{~cm}$. A gravid female was observed in June 2007 with 5 embryos. For $D$. americana a total of 224 specimens were analyzed; $40 \%$ of these had not reached sexual maturity; females showed higher sizes (21.2-164 $\mathrm{cm})$ than males $(35-147 \mathrm{~cm})$ and also a higher size at maturity $\left(L_{50}\right.$ : females: $64 \mathrm{~cm}$, males: $\left.56 \mathrm{~cm}\right)$, but all specimens were mature above $70 \mathrm{~cm}$. Differences in the sex ratio and gravid females were observed only for August 2006 and March 2007, with a highest fecundity of 5 embryos. We recommend setting minimum catch lengths and periods of closure of the fishery.
\end{abstract}

Key words: Elasmobranchii, Chondrichthyes, southern stingray, longnose stingray, Caribbean

\begin{abstract}
Resumen.- En Venezuela, las rayas Dasyatis guttata y D. americana son consideradas comunes y con importancia comercial. A pesar de existir pesquerías dirigidas a capturarlas, existen pocos estudios sobre su biología, siendo ambas catalogadas en la IUCN con datos deficientes. Por esto, el presente estudio planteó analizar las capturas de la pesquería artesanal de la Isla de Margarita, con la finalidad de aportar datos biológicos que contribuyan a su manejo pesquero. Para ello entre enero 2006 y diciembre 2007, se visitaron una vez por semana 3 comunidades pesqueras y 2 mercados de venta de pescado. A cada ejemplar se le midió la longitud del ancho del disco, se determinó el sexo y estadio de madurez. Un total de 355 ejemplares de $D$. guttata demostraron que las hembras $(32-173 \mathrm{~cm})$ alcanzan longitudes superiores que los machos (28,6$113 \mathrm{~cm}$ ); el $35 \%$ de las capturas correspondió a individuos inmaduros; se encontraron diferencias en la proporción sexual pero no en las tallas de madurez $\left(\mathrm{L}_{50}\right.$ para ambos sexos: $56 \mathrm{~cm}$ ); todos los individuos se encontraron maduros a partir de $78 \mathrm{~cm}$; sólo se observó una hembra grávida (junio 2007), con fecundidad de 5 embriones. Para D. americana se analizaron 224 ejemplares, de los cuales el 40\% eran inmaduros; las hembras mostraron longitudes superiores (21,2-164 cm) que los machos $(35-147 \mathrm{~cm})$, y maduran a mayores longitudes $\left(\mathrm{L}_{50}: \mathrm{H}: 64 \mathrm{~cm}\right.$; M: $\left.56 \mathrm{~cm}\right)$; todos los individuos se encontraron maduros a partir de $70 \mathrm{~cm}$; hubo diferencias en la proporción sexual y sólo se observaron hembras grávidas para agosto 2006 y marzo 2007, siendo la fecundidad máxima uterina de 5 embriones. Se recomienda establecer longitudes mínimas de captura y periodos de veda.
\end{abstract}

Palabras clave: Elasmobranchii, Chondrichthyes, raya hocicona, raya látigo, Mar Caribe

\section{INTRODUCCIÓN}

La raya hocicona, Dasyatis guttata (Bloch \& Schneider, 1801) y la raya látigo, Dasyatis americana Hildebrand \& Schoreder, 1928, son consideradas comunes en aguas de la plataforma continental de Venezuela (Cervigón \& Alcalá 1999). La carne de ambas especies es bastante apreciada y frecuentemente es utilizada como sustituta en la 
preparación de platos típicos regionales, cuando hay escasez de pequeños cazones (Rhizoprionodon spp. y Mustelus spp.) o del chucho pintado (Aetobatus narinari).

Se ha reportado que las rayas de la familia Dasyatidae no poseen amenaza directa de explotación, siendo capturadas únicamente de manera incidental en algunas pesquerías (Graham et al. 2009). Sin embargo, en Venezuela, por ser una especie de considerable valor comercial, existen pesquerías dirigidas a su captura, e incluso, existe un arte de pesca exclusivo para su captura, conocido como filete o tren rayero, el cual utiliza una red de enmalle de fondo. Adicionalmente, son también fauna acompañante y parte importante de las capturas de otras pesquerías, como el palangre de fondo y el arrastre artesanal.

Ambas especies son catalogadas 'con Datos Deficientes' en la lista de especies amenazadas de la Unión Internacional para la Conservación de la Naturaleza (UICN) (Rosa \& Furtado 2004, Grubbs et al. 2006). No obstante, D. americana es considerada 'Vulnerable' en Brasil (Buckup et al. 2000) y se ha recomendado monitorear sus capturas en Venezuela (Grubbs et al. 2006). Para D. guttata se conoce que la presión de pesca ha aumentado en aguas brasileras, siendo necesaria su evaluación poblacional en países vecinos, para establecer con mayor claridad su estatus de conservación (Rosa \& Furtado 2004).

El objetivo del presente estudio fue generar información biológica en cuanto a las estructuras de tamaños y aspectos reproductivos de $D$. americana y $D$. guttata capturadas por la flota pesquera artesanal de la isla de Margarita, con la finalidad de generar información de base que contribuya a procesos de manejo y conservación.

\section{MATERIALES Y MÉTODOS}

\section{ÁREA DE ESTUDIO}

La isla de Margarita se ubica en la región nororiental de

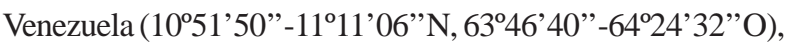
donde existen masas de agua moderadamente fértiles (Gómez 1996), debido a la acción del viento que provoca la surgencia de aguas frías y ricas en nutrientes durante los primeros meses del año (Castellanos et al. 2000). Otros factores propician esta fertilidad durante todo el año, tales como las descargas de materia orgánica de ríos e.g., Orinoco y Amazonas, la presencia de lagunas costeras y la influencia de ondas internas e islas que causan enriquecimientos locales (Gómez 1996). Particularmente, al norte de la Isla de Margarita han sido identificados focos de surgencia, y aunque su efecto es local, provocan una importante dispersión de aguas frías hacia el norte (Castellanos et al. 2000). En conjunto, estos eventos propician la existencia permanente de recursos pesqueros de importancia económica en la zona (Ginés et al. 1972, Gómez 1996, Castellanos et al. 2000, Freón \& Mendoza 2003).

En la Isla de Margarita se concentran 10.162 pescadores artesanales activos, distribuidos en unas 59 comunidades pesqueras y 8 refugios de pesca (González et al. 2006). Los ejemplares de raya analizados provinieron de embarcaciones de madera tipo peñero ( $<12 \mathrm{~m}$ de eslora), propulsadas con motores fuera de borda a gasolina y que utilizan redes de enmalle de fondo (tren rayero), cuyas dimensiones varían dependiendo de las preferencias y presupuesto del pescador (longitud: entre 300 y 1000 m; altura: entre 7 y $10 \mathrm{~m}$; luz de malla: 12, 14 ó 16 pulgadas; hilo: 24, 27, 30 ó 36). Descripciones detalladas de las embarcaciones y artes de pesca, son reseñadas por Méndez-Arocha (1963), Ginés et al. (1972), Iriarte (1997) y González et al. (2006).

\section{Trabajo de campo}

Se realizaron muestreos entre enero 2006 y diciembre 2007, una vez por semana, en 3 puertos pesqueros (Juan Griego, La Pared y El Tirano) y 2 mercados de venta de pescado (Los Cocos y Conejeros). Los especímenes fueron identificados utilizando las claves taxonómicas aportadas por Cervigón \& Alcalá (1999). A cada individuo se le midió el ancho del disco (Ad), definido como la distancia comprendida entre las puntas de las aletas pectorales. Adicionalmente, un individuo de cada especie fue catalogado en la 'Colección Ictiológica de la Escuela de Ciencias Aplicadas del Mar' (CI-ECAM) de la Universidad de Oriente, Boca de Río, Isla de Margarita, Venezuela.

\section{Análisis de datos}

Para establecer la proporción sexual, los sexos se diferenciaron por la presencia de claspers en machos y por su ausencia en las hembras. Para evaluar diferencias en la proporción sexual mensual de machos y hembras, se aplicó una prueba de Chi-cuadrado $\left(\chi^{2}\right)$, considerando la corrección de Yates (Zar 1996):

$$
\chi_{i}^{2}=\left(\left|\mathrm{f}_{o_{i}}-\mathrm{f}_{s_{i}}\right|-1\right)^{2} / N_{i}
$$

donde, $\mathrm{f}_{\mathrm{o} i}$ es la frecuencia de machos en el mes $i ; \mathrm{f}_{\mathrm{a} i}$ es frecuencia de hembras en el mes $i$ y $N_{i}$ es frecuencia de machos y hembras en el mes $i$. 
A fin de comparar las longitudes de machos y hembras para ambas especies, se realizó una prueba de suma de rangos Mann-Whitney, ya que los datos no cumplieron los supuestos de normalidad (Kolmogorov-Smirnov, $P<$ $0,001)$.

La madurez sexual de los machos fue verificada a partir del grado de calcificación de los claspers; aquellos individuos que los presentaron fuertemente calcificados, con fácil rotación en la base y extremo distal extensible, fueron considerados como individuos maduros (Conrath 2004).

Debido a la rapidez en que los individuos son cortados para su venta al llegar a los puertos, la madurez de las hembras fue identificada a través de la observación macroscópica del aparato reproductivo utilizando sólo 3 categorías: inmaduras, maduras o grávidas. Dependiendo de la presencia o ausencia de útero y ovario claramente desarrollados, fueron consideradas maduras o inmaduras, respectivamente. Las hembras grávidas presentaron embriones en el útero, los cuales se les midió el ancho del disco y fueron contados para estimar la fecundidad uterina (Conrath 2004).
Se estimó la talla media de madurez sexual para los sexos por separado, para lo cual se determinó la proporción de organismos maduros para cada intervalo de talla, y se estimó la proporción de maduros predicha para cada talla mediante la función logística, ajustando mediante el método de mínimos cuadrados:

$$
M_{f}=\frac{1}{1+e^{-a\left(L_{i}-b\right)}}
$$

donde $M_{f}$ es la fracción de individuos maduros; el parámetro $a$ representa el cambio en la pendiente de $M_{f}$ como función de la longitud del ancho del disco $\left(L_{i}\right)$, y $b$ representa la longitud a la cual el $50 \%$ de los individuos están maduros $\left(L_{50}\right)$. Los intervalos de confianza de $a$ y $b$ se calcularon utilizando 2500 remuestreos de los residuos (bootstrap no paramétrico).

Una vez obtenidas las tallas de madurez para machos y hembras por separado, se utilizó como criterio los intervalos de confianza de ambos estimados, para definir si existen o no diferencias entre las estimaciones de longitudes de madurez de ambos sexos, y en caso de no

Tabla 1. Aspectos biológicos de las rayas Dasyatis guttata y D. americana capturadas por la flota artesanal de la Isla de Margarita, Venezuela / Biological aspects of rays Dasyatis guttata and D. americana caught by the artisanal fishery in Margarita Island, Venezuela

\begin{tabular}{lcc}
\hline & Dasyatis guttata & Dasyatis americana \\
\hline Nombre común & Raya hocicona & Raya látigo \\
Individuos analizados & 355 & 224 \\
Intervalo de tamaño hembras (cm de Ad) & $32-173$ & $21,2-164$ \\
Intervalo de tamaño machos (cm de Ad) & $28,6-113$ & $35-147$ \\
$\%$ de machos (M) & 35 & 41 \\
$\%$ de hembras (H) & 65 & 59 \\
Proporción sexual (H:M) & $1,9: 1$ & $1,5: 1$ \\
Talla media de madurez (H) (cm de Ad) & $56,4(95 \% \mathrm{CI}=56,3-56,8)$ & $64(95 \% \mathrm{CI}=62,9-65)$ \\
Talla media de madurez $(\mathrm{M})(\mathrm{cm}$ de Ad) & $56,4(95 \% \mathrm{CI}=54,6-58,5)$ & $56,3(95 \% \mathrm{CI}=55,5-56,9)$ \\
Talla de madurez ambos sexos (cm de Ad) & $56(95 \% \mathrm{CI}=55,9-56,2)$ & $\neq$ \\
Talla media de captura \pm D.S (cm de Ad) & $69,2 \pm 24,1$ & $71 \pm 27,5$ \\
$\%$ de machos inmaduros & 55,3 & 36 \\
$\%$ hembras inmaduras & 24 & 41 \\
Hembra grávida de menor tamaño (cm de Ad) & 125 & 120 \\
Fecundidad máxima uterina & 5 & 5 \\
Longitud máxima embrión (cm de Ad) & 10 & 17,7 \\
Longitud mínima juvenil libre (cm de Ad) & 28,6 & 21,2 \\
\hline
\end{tabular}


existir, se determinó una única talla madurez sexual para ambos sexos, con la finalidad de proveer una sola longitud de madurez que contribuya a facilitar el trabajo a las gerencias de ordenación pesquera y pescadores.

\section{Resultados}

\section{DASYATIS GUTTATA}

Se analizaron 355 individuos, 232 hembras y 123 machos, encontrándose diferencias significativas en la proporción sexual $\left(\chi^{2}=31,6 ; P<0,05\right)$ (Tabla 1$)$.

Tomando como criterio los intervalos de confianza, no se detectaron diferencias entre las tallas media de madurez sexual para los sexos por separado, por lo que se calculó una sola longitud media de madurez ubicada en $56 \mathrm{~cm}$ (Tabla 1, Fig. 1). Sin embargo, el $100 \%$ de machos y hembras se encontraron maduros por encima de los 78 $\mathrm{cm}$, y sólo se observaron hembras grávidas por encima de $120 \mathrm{~cm}$.

Las hembras alcanzaron mayores longitudes que los machos (Mann-Whitney, $\mathrm{U}=15266, P<0,001$ ) (Fig. 2). En general, el $65 \%$ de la capturas correspondió a individuos sexualmente maduros. Las hembras maduras representaron el $75 \%$ de la muestra analizada, y estuvieron presentes prácticamente en todos los meses, excepto en mayo 2006. Se observó un menor porcentaje de machos maduros (45\%) respecto a los inmaduros (Fig. 3).

Una sola hembra grávida fue observada en junio 2007 (Fig. 3). La fecundidad máxima fue de 5 embriones, que alcanzaron una longitud máxima de $10 \mathrm{~cm}(\bar{X}: 9,4 \pm 0,5$ $\mathrm{cm})$, sin embargo, todavía presentaban saco vitelino y coloración crema transparente, por lo que no se consideraron cercanos a la longitud de nacimiento.

\section{DASYATIS AMERICANA}

Se analizaron 224 individuos, 133 hembras y 91 machos. También se encontraron diferencias significativas en la proporción sexual total $\left(\chi^{2}=7,7 ; P<0,05\right)$ (Tabla 1). El $60 \%$ de los ejemplares analizados fueron individuos maduros.

Las hembras alcanzaron longitudes mayores que los machos (Mann-Whitney, $\mathrm{U}=8839, P=0,003$ ) (Fig. 2); además se evidenciaron diferencias entre las estimaciones de la talla de madurez sexual para machos y hembras, por lo que se presentan por separado (Tabla 1, Fig. 1). El $100 \%$ de los organismos se encontraron maduros a longitudes superiores a $70 \mathrm{~cm}$.

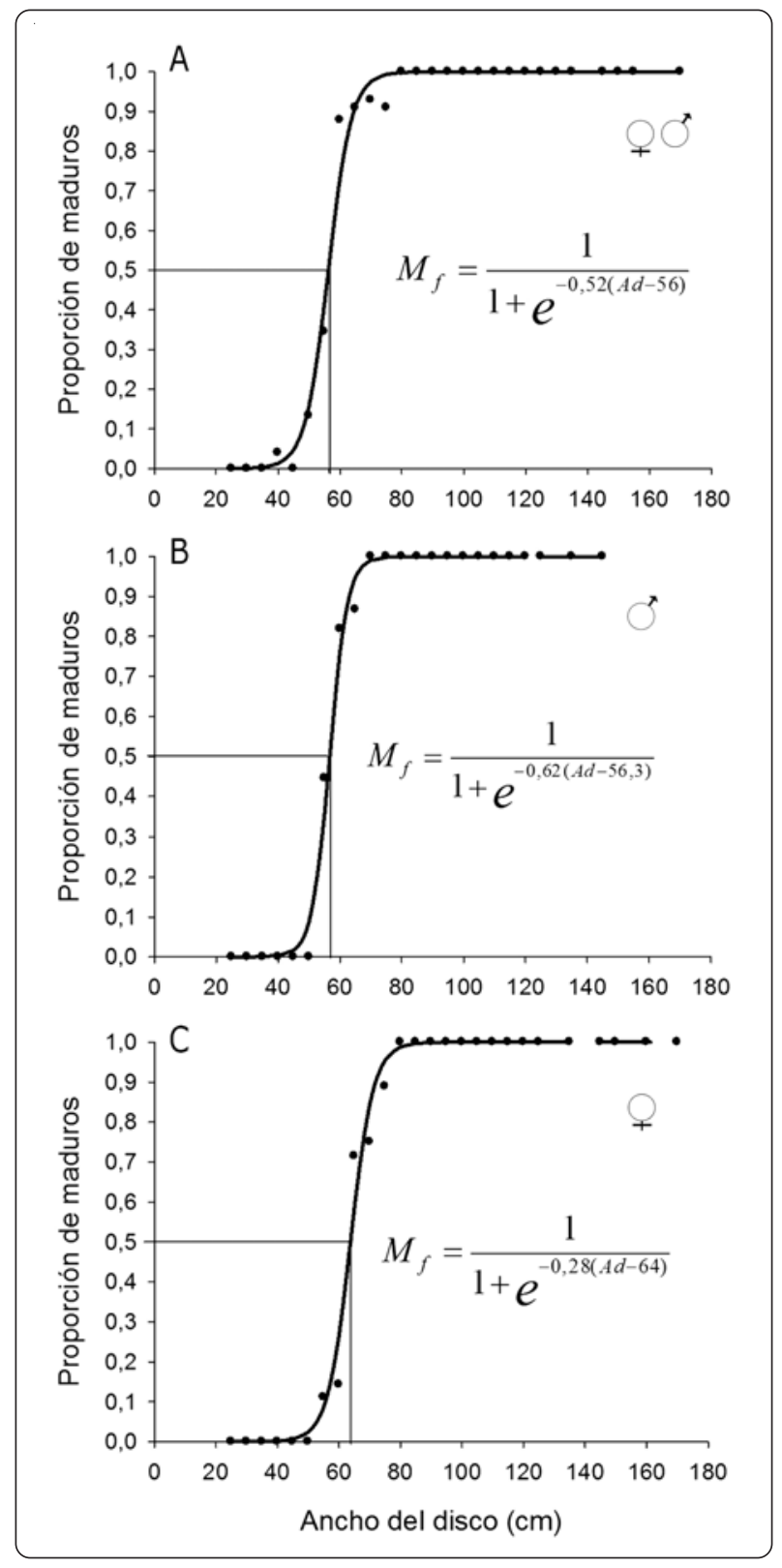

Figura 1. Función logística de la talla media de madurez. A) Dasyatis guttata ambos sexos; B-C) Dasyatis americana machos y hembras capturadas por la pesquería artesanal de isla de Margarita, Venezuela / Length at maturity logistic functions. A) Dasyatis guttata both sex; B-C) Dasyatis americana males and females caught by the artisanal fishery of Margarita Island, Venezuela

Sólo fueron observadas 2 hembras grávidas con longitudes superiores a $120 \mathrm{~cm}$, en agosto 2006 y marzo 2007 (Fig. 4). La fecundidad máxima fue de 5 embriones, cuya longitud máxima fue de $17,7 \mathrm{~cm}(\bar{X}: 11,7 \pm 3,03)$, mientras que el ejemplar de vida libre de menor longitud alcanzó los 21,2 cm. 


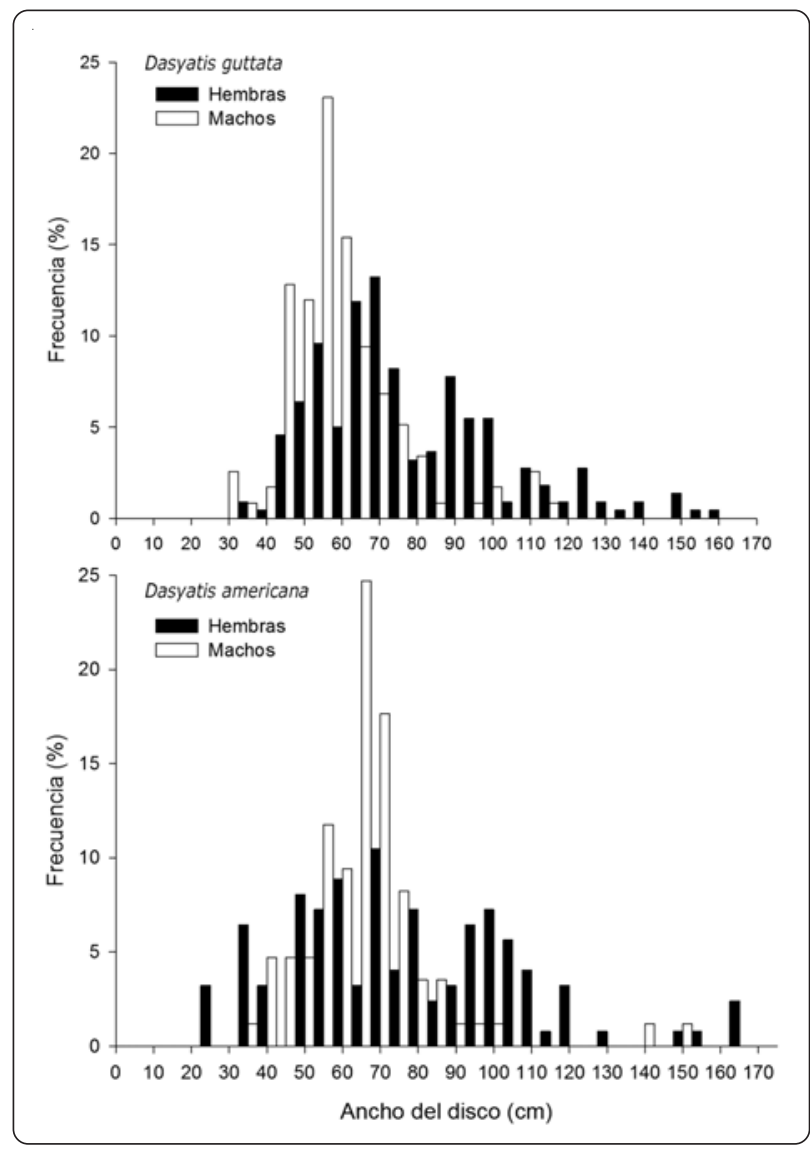

Figura 2. Estructura de tallas de Dasyatis guttata y D. americana, capturadas por la flota pesquera artesanal de isla de Margarita, Venezuela / Length structure of Dasyatis guttata y D. americana, caught by the artisanal fishery of Margarita Island, Venezuela

\section{Discusión}

En Venezuela, D. americana ha sido considerada la especie más abundante del género (Cervigón \& Alcalá 1999). Sin embargo, los resultados de este trabajo y los aportados por Cordovés et al. (2009) muestran que $D$. guttata es más abundante en las capturas actuales de Venezuela, así como en otras pesquerías artesanales del mar Caribe (Grijalba-Bendeck et al. 2007, Mojica-Moncada 2007, Moreno et al. 2008, Grijalba-Bendeck et al. 2012).

La longitud máxima observada para $D$. guttata (173 $\mathrm{cm})$, es inferior a la de $200 \mathrm{~cm}$ reportada previamente por Cervigón \& Alcalá (1999); la misma condición fue observada para $D$. americana, con una longitud máxima de $164 \mathrm{~cm}$ para este estudio y el Golfo de México (RamírezMosqueda et al. 2012), que resulta inferior a la reportada por Cervigón \& Alcalá (1999) de 174 cm. Este fenómeno podría ser atribuido a diferencias temporales, esfuerzos de muestreo y artes de pesca. Sin embargo, la disminución de tallas es señalada como el inicio de la sobreexplotación (Linton \& Warner 2003).

Con respecto a Dasyatis guttata, en todo el periodo de estudio, las capturas estuvieron compuestas en su mayoría por individuos maduros, lo cual es similar a lo observado en Colombia (Mojica-Moncada 2007, GrijalbaBendeck et al. 2012). Sin embargo, en otra pesquería dirigida a estas rayas en la Isla de Cubagua, detectaron altos porcentajes de individuos inmaduros para ambos sexos (Cordovés et al. 2009).

La observación de hembras con longitudes mayores a los machos, coincide con lo observado por Cordovés et al. (2009) para la pesquería de Cubagua, y por MojicaMoncada (2007) en Colombia. Estudios previos indican que las hembras alcanzan la madurez sexual a longitudes superiores que los machos $(\mathrm{H}=53,9 \mathrm{~cm}, \mathrm{M}=39 \mathrm{~cm}$, MojicaMoncada (2007); $\mathrm{H}=64,1 \mathrm{~cm}, \mathrm{M}=54,7 \mathrm{~cm}$, Salas \& Tejeda (2009); $\mathrm{H}=62,3 \mathrm{~cm}, \mathrm{M}=57,5 \mathrm{~cm}$, Cordovés et al. (2009). En este estudio la talla de madurez sexual de los machos fue igual que la calculada para las hembras y los intervalos de confianza de dichos estimados permiten asumir que no hay diferencias significativas, lo que podría facilitar el manejo pesquero de la especie. Las diferencias observadas con respecto a otros estudios, pueden ser atribuidas a variaciones entre las áreas de pesca, métodos de evaluación, número de ejemplares analizados y a que pueden ser comunes las diferencias biológicas entre poblaciones o subpoblaciones de elasmobranquios separadas geográficamente (Henningsen et al. 2004).

La proporción sexual concuerda con lo reportado por Thorson (1983) y Cordovés et al. (2009), las hembras son más numerosas que los machos. Sin embargo, para Colombia, Mojica-Moncada (2007) encuentra iguales proporciones sexuales.

La única hembra grávida se encontró en junio; en Colombia, se han reportado en marzo, junio y octubre (Mojica-Moncada 2007). Así mismo, una fecundidad de 5 embriones, se asemeja a lo observado para Colombia, con un promedio de 6 embriones (Mojica-Moncada 2007). La longitud máxima de embriones registrada, es menor a la longitud cercana al nacimiento $(15 \mathrm{~cm})$, sugerida por Bigelow \& Schoreder (1953); los embriones observados no estaban totalmente desarrollados, presentando reservas de vitelo. En función del ejemplar de vida libre con menor longitud conocida (15,4 cm) (Mojica-Moncada 2007) y de los embriones de mayor longitud reportados, la especie probablemente tenga un intervalo de talla cercano al nacimiento de unos 15 a $17 \mathrm{~cm}$. La escasez de 


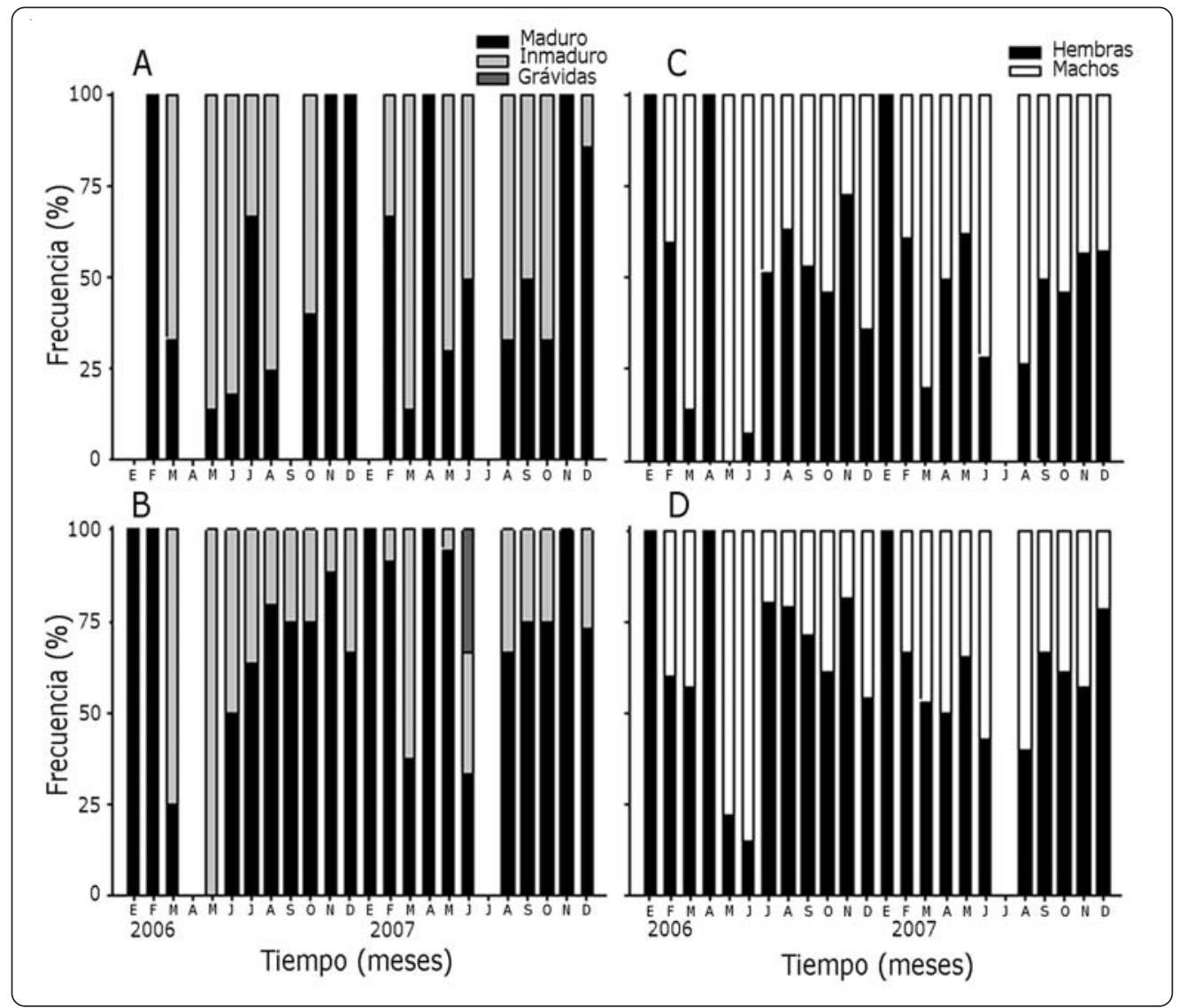

Figura 3. Estadios de madurez en Dasyatis guttata. A) machos, B) hembras, C) proporción sexual de ejemplares maduros y D) proporción sexual general de los ejemplares capturados por la pesquería artesanal de Isla Margarita, Venezuela / Dasyatis guttata maturity stages. A) males, B) females, C) sexual proportion of mature organisms and $\mathrm{D})$ general sexual proportion of the specimens caught by the artisanal fishery in Isla Margarita, Venezuela

embriones impide calcular un posible periodo de gestación, aunque ha sido señalado que puede estar entre unos 5 y 6 meses (Mojica-Moncada 2007).

En relación a Dasyatis americana, un dimorfismo sexual, con hembras que alcanzan longitudes mayores que los machos, coincide con lo reportado en estudios previos (Salas \& Tejeda 2009, Henningsen \& Leaf 2010, Ramírez-Mosqueda et al. 2012); y aunque han sido estimadas longitudes asintóticas de 150,9 cm para hembras y 67,4 cm para machos (Henningsen \& Leaf 2010), en este estudio 34 individuos superaron estas longitudes.

De igual manera, el hecho que las hembras maduren a longitudes superiores que los machos, ha sido previamente reportado por Henningsen \& Leaf (2010; H $=75-80 \mathrm{~cm}, \mathrm{M}=52 \mathrm{~cm}$ ) y por Ramírez-Mosqueda et al. (2012; $\mathrm{H}=76,4 \mathrm{~cm}, \mathrm{M}=51,7 \mathrm{~cm}$ ).
En Brasil se han reportado resultados similares respecto a la proporción sexual, siendo también mayor el número de hembras (McEachran \& de Carvalho 2002). Sin embargo, se han descrito proporciones sexuales iguales en neonatos en cautiverio (Henningsen 2000, Henningsen \& Leaf 2010), por lo que las diferencias observadas en el presente estudio podrían indicar segregación sexual en adultos.

La fecundidad de 5 embriones en este estudio, se asemeja a lo indicado por Henningsen (2000) (4 embriones) y al intervalo de 2 a 7 señalado por RamírezMosqueda (2012). De igual forma, el intervalo de longitud cercano al nacimiento entre 17 y $19 \mathrm{~cm}$ (McEachran \& de Carvalho 2002), es congruente con la longitud máxima de embrión (17,7 cm), y con el ejemplar de vida libre de menor longitud (21,2 cm). No obstante, Ramírez-Mosqueda et al. (2012) señalan un intervalo de longitud de nacimiento de 23 a $34 \mathrm{~cm}$. El escaso número de hembras grávidas, y 


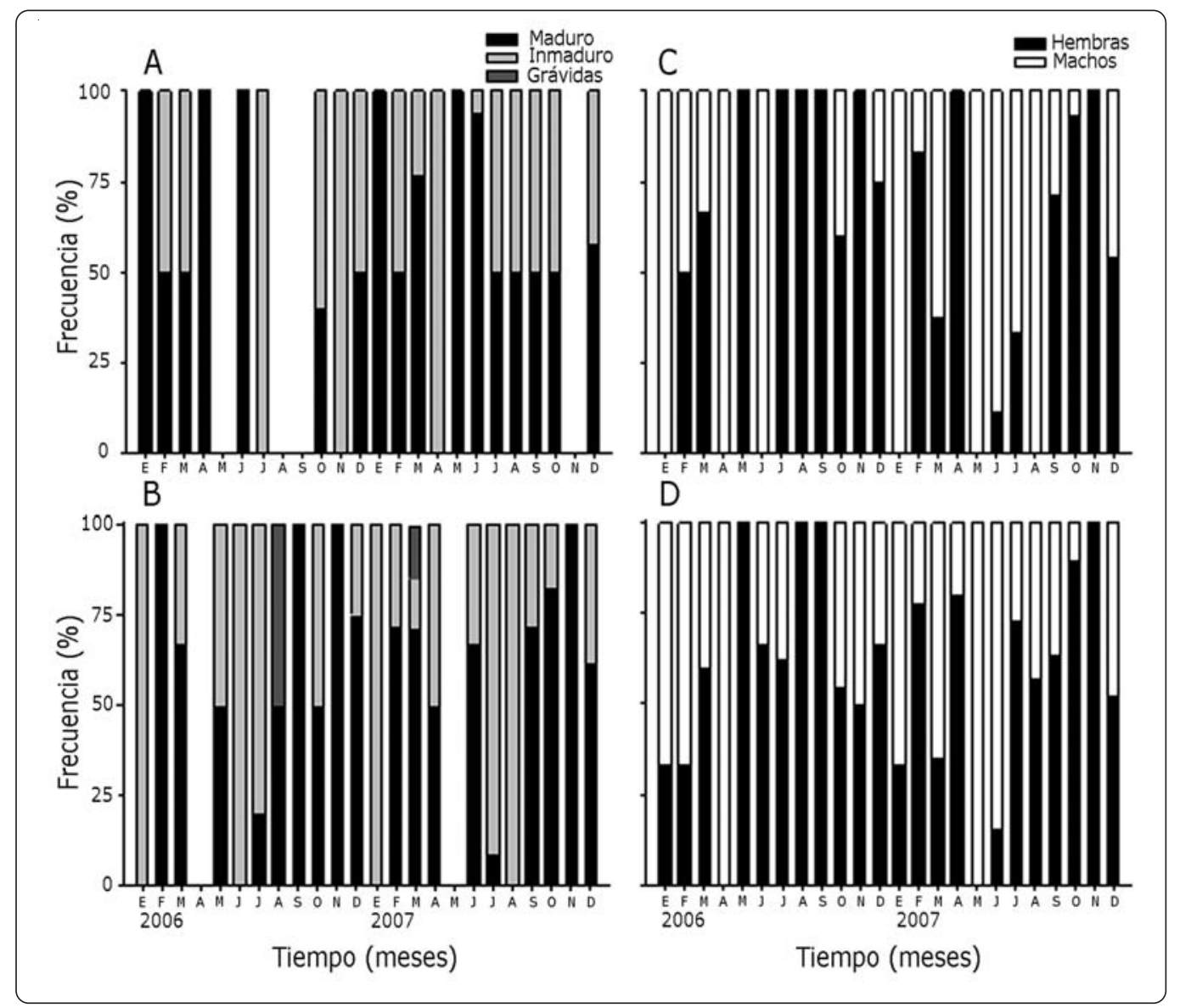

Figura 4. Estadios de madurez en Dasyatis americana. A) machos, B) hembras, C) proporción sexual de ejemplares maduros y D) proporción sexual general de los ejemplares capturados por la pesquería artesanal de Isla Margarita, Venezuela / Dasyatis americana maturity stages. A) males, B) females, C) sexual proportion of mature organisms and D) general sexual proportion of the specimens caught by the artisanal fishery in Isla Margarita, Venezuela

de embriones analizados, impide indagar acerca del periodo de gestación; sin embargo, Henningsen (2000) ha sugerido un periodo de gestación de entre 4,5 y 7,5 meses. Estudios recientes en el Golfo de México sugieren que se reproducen anualmente con un periodo de gestación entre 7 y 8 meses (Ramírez-Mosqueda et al. 2012), aunque Yokota \& Lessa (2007) reportan, para el noreste de Brasil, que la especie posee un ciclo reproductivo bianual.

El predominio de individuos maduros en las capturas, es un hecho que coincide con lo observado en pesquerías artesanales del Golfo de México (Ramírez-Mosqueda et al. 2012), sin embargo, para Venezuela, un $40 \%$ de capturas de individuos inmaduros puede representar un alto impacto para el futuro de la pesquería.
En las estadísticas oficiales venezolanas, los datos de capturas de ambas especies están unidos en un solo ítem denominado rayas varias, en los que se evidencia un declive progresivo de las capturas. A pesar de que en Venezuela no existen leyes dirigidas a regular las capturas de estas especies, la prohibición de la pesca de arrastre industrial (Art. 23, Ley de Pesca y Acuicultura 2008 ) puede considerarse una medida que contribuye a su conservación. No obstante, resulta necesario establecer una talla mínima de captura, en función de la madurez sexual, y considerar una veda de al menos 4 meses por año, en función de sus posibles máximos picos reproductivos, a fin de contribuir a que sus capturas se mantengan en el tiempo.

\footnotetext{
1 Ley de Pesca y Acuicultura. 2008. Artículo №23. Decreto № 5930 Publicado en Gaceta Oficial de la República Bolivariana de Venezuela №5877 de 14 de marzo de 2008.
} 
En el Caribe, el turismo enfocado a la observación de rayas en aguas de poca profundidad ha sido un incentivo para conservar sus poblaciones (Grubbs et al. 2006). Promover la protección de áreas de reproducción y crianza son necesarias para la supervivencia a largo plazo de estas especies (Rosa \& Furtado 2004, Grubbs et al. 2006).

\section{Agradecimientos}

A todos los pescadores y vendedores de pescado que gentilmente permitieron analizar los ejemplares. A Asdrúbal Lárez y al Instituto Nacional de Investigaciones Agrícolas (INIA) por la colaboración en la logística de muestreos. A los revisores anónimos por ayudar a mejorar el manuscrito.

\section{LITERATURA CITADA}

Bigelow H \& W Schroeder. 1953. Fishes of the Western North Atlantic. Part 2: Sawfishes, guitarfishes, skates and rays; chimaeroids, 576 pp. Yale University, New Haven.

Buckup P, G Nunan, U Gómez, W Costa \& O Gadig. 2000. Peixes. Em: Di Maio F \& M Silva (eds). Espécies ameaçadas de extinçao no Município do Rio de Janeiro: flora e fauna, 52-60 pp. Secretaria Municipal de Meio Ambiente, Rio de Janeiro.

Castellanos P, R Varela \& F Muller-Karger. 2000. Descripción de las áreas de surgencia al sur del Mar Caribe examinadas con el sensor infrarrojo AVHRR. Memoria de la Fundación La Salle de Ciencias Naturales 154: 55-76.

Cervigón F \& AAlcalá. 1999. Los peces marinos de Venezuela 5: 1-230. Fundación Museo del Mar, Caracas.

Conrath C. 2004. Reproductive biology. In: Musick J \& R Bonfill (eds). Elasmobranch fisheries management techniques, pp. 133-164, APEC Secretariat, Singapore.

Cordovés M, E Ron \& R Tavares. 2009. Composición de las capturas comerciales de Raya Látigo-Hocicona, Dasyatis guttata (Bloch \& Schneider, 1801) procedentes de la pesca artesanal en la Isla de Cubagua, Venezuela. Proceedings of the Gulf and Caribbean Fisheries Institute 62: 567-570.

Freón P \& J Mendoza. 2003. La sardina (Sardinella aurita), su medio ambiente y explotación en el oriente de Venezuela, 549 pp. Institut de Recherche pour le Développement, Collection Colloques et Séminaires, Paris.

Ginés H, C Angell, M Méndez, G Rodríguez, G Febres, R Gómez, J Rubio, G Pastor \& J Otaola. 1972. Carta pesquera de Venezuela. Áreas del nororiente y Guayana, Monografía 16: 1-327. Fundación La Salle de Ciencias Naturales, Caracas.

Gómez A. 1996. Causas de la fertilidad marina en el nororiente de Venezuela. Interciencia 21(3): 140-146.
González L, N Eslava \& F Guevara. 2006. Catálogo de la pesca artesanal del Estado Nueva Esparta, Venezuela, 222 pp. Instituto de Investigaciones Científicas, Universidad de Oriente, Cumaná.

Graham L, B Murphy \& D Hata. 2009. Using species composition data from a trawl survey to determine potential bycatch of the commercial trawl fishery for horseshoe crab Limulus polyphemus in the Middle Atlantic Bight. North American Journal of Fisheries Management 29: 478-487.

Grijalba-Bendeck M, C Polo-Silva \& A Acero. 2007. Una aproximación a la abundancia de los batoideos capturados artesanalmente en Santa Marta. Boletín de Investigaciones Marinas y Costeras 36: 251-268.

Grijalba-Bendeck M, C Polo-Silva, K Acevedo, F Moreno \& D Mojica. 2012. Aspectos tróficos y reproductivos de algunos batoideos capturados en Santa Marta, Mar Caribe de Colombia. Latin American Journal of Aquatic Research 40(2): 300-315.

Grubbs D, F Snelson, A Piercy, R Rosa \& M Furtado. 2006. Dasyatis americana. In: IUCN (ed). IUCN Red list of threatened species, Version 2011.2. International Union for Conservation of Nature. <www.iucnredlist.org>

Henningsen A. 2000. Notes on reproduction in the southern stingray, Dasyatis americana (Chondrichthyes: Dasyatidae), in a captive environment. Copeia 2000: 826828.

Henningsen A \& R Leaf. 2010. Observations on the captive biology of the southern stingray. Transaction American of the Fisheries Society 139: 783-791.

Henningsen A, M Smale, R Garner \& N Kinnunen. 2004. Reproduction, embryonic development, and reproductive physiology of elasmobranchs. In: Smith M, D Warmolts, D Thoney \& R Hueter (eds). Elasmobranch husbandry manual: captive care of sharks, rays and their relatives, pp. 227-236. Ohio Biological Survey, Columbus.

Iriarte L. 1997. Embarcaciones, artes y métodos de pesca del estado Nueva Esparta, Monografía 42: 1-349. Fundación La Salle de Ciencias Naturales, Caracas.

Linton D \& G Warner. 2003. Biological indicators in the Caribbean coastal zone and their role in integrated coastal management. Ocean \& Coastal Management 46: 261-276.

McEachran J \& M de Carvalho. 2002. Dasyatidae. In: Carpenter K (ed). The living marine resources of the Western Central Atlantic, Volume 1. Introduction, molluscs, crustaceans, hagfishes, sharks, batoid fishes and chimaeras, pp. 562-571. FAO Species Identification Guides for Fishery Purposes, Roma.

Méndez-Arocha A. 1963. La pesca en Margarita. Monografía 7: 1-267. Estación de Investigaciones Marinas de Margarita, Fundación La Salle de Ciencias Naturales, Editorial Vizcaya, Bilbao. 
Mojica-Moncada D. 2007. Bioecología de la raya látigo Dasyatis guttata capturada con artes de pesca artesanal en Don Jaca, Santa Marta-Caribe colombiano. Tesis de grado, Universidad Jorge Tadeo Lozano, Santa Marta, 96 pp.

Moreno F, K Acevedo \& J Paramo. 2008. Aportes a la biología de tiburones y rayas demersales en la región norte del Caribe Colombiano. Acta Biológica Colombiana 13: 121-130.

Ramírez-Mosqueda E, J Pérez-Jiménez \& M MendozaCarranza. 2012. Reproductive parameters of the southern stingray Dasyatis americana in southern gulf of Mexico. Latin American Journal of Aquatic Research 40(2): 335344.

Rosa R \& M Furtado. 2004. Dasyatis guttata. In: IUCN (ed). IUCN Red list of threatened species, Version 2011.2. International Union for Conservation of Nature. $<$ www.iucnredlist.org >
Salas S \& K Tejeda. 2009. Aspectos biológico-pesqueros de tres especies de la familia Dasyatidae aprovechadas artesanalmente entre Tasajera y Jorará, zona costera del Magdalena, (Agosto 2008-Febrero2009). Tesis de Grado, Universidad del Magdalena, Santa Marta, 95 pp.

Thorson T. 1983. Observations on the morphology, ecology, and life history of the euryhaline stingray, Dasyatis guttata (Bloch and Schneider) 1801. Acta Biológica Venezuelica 11: 95-125.

Yokota L \& R Lessa. 2007. Reproductive biology of three ray species: Gymnura micrura (Bloch \& Schneider, 1801), Dasyatis guttata (Bloch \& Schneider, 1801) and Dasyatis marianae Gomes, Rosa \& Gadig, 2000, caught by artisanal fisheries in northeastern Brazil. Cahiers de Biologie Marine 48: 249-257.

Zar J. 1996. Biostatistical analysis, 662 pp. Prentice Hall, Upper Saddle River.

Recibido el 15 de diciembre de 2012 y aceptado el 2 de julio de 2013

Editor: Claudia Bustos 